In cases of suppuration of the internal ear, I do not apply a graft, as the object of the operation is to obtain free drainage, and $I$ think that the application of a graft is very liable to defeat this object.

\title{
NOTES ON THE RADICAL MASTOID OPERATION.'
}

\author{
By J. S. Fraser, M.B., F.R.C.S.E.
}

(From the Ear and Throat Department of the Royal Infirmary, Edinburgh, under the charge of A. Logan 'TURNer, M.D., F.R.C.S.E., F.R.S.E.)

IT has always appeared to the writer that we expect a good deal from Nature when we perform the radical mastoid operation. The Eustachian tube is open and we want it to close. Secondly, the external meatus is separated from the tympanum by the drumbead and external attic wall, and yet after we have removed the remains of the membrane along with the bony ledge we expect them not to reform. Thirdly, the external meatus is separated from the mastoid antrum by the bony posterior meatal wall which we take away, knowing that there is a great tendency to its reproduction. 'Thus we want to close a tube which often persists in remaining open and from which a mucoid discharge spreads over the inner tympanic wall. We also wish to maintain the patency of two artificial openings which tend to close.

In common with many other otologists the writer begins the radical operation by making a semi-circular incision in the hair margin, curving forward below to the tip of the mastoid (Fig. 1), because when the wound is stitched up at the end of the operation the line of incision lies over the posterior part of the mastoid process, which does not require removal except in cases where a sinus thrombosis or an extradural abscess in the posterior fossa have to be dealt with. The flap thus outlined is dissected forward subcutaneously till the region of the retro-auricular groove is reached, when an incision is made through the periosteum down to the bone. In this way a valvular wound is obtained and the line of incision is not "in the air" as is the case when the cut is made in the retro-auricular groove. In at least nineteen cases out of twenty the wound heals by fist intention, without a drop of pus, and in the twentieth there is at most a stitch abscess or two. The writer, however, does not deny that equally good results may be obtained by other methods.

1 Contribution to Discussion at the Otological Section of the Royal Society of Medicine. See p. 95. 
With regard to the gouges employed in the removal of bone, it has always appeared to the writer that-the masonic craft being an exceedingly old one-the nearer the otologist could get to the gouge, or chisel, of the stone-masons the better. The Vienna pattern of gouge seems to be the nearest approach. This instrument has a large, rounded, proximal end which projects from the shank so that the operator can get the end of his left thumb up against the projection and thus prevent the instrument from slipping and injuring the facial nerve or labyrinth, when the

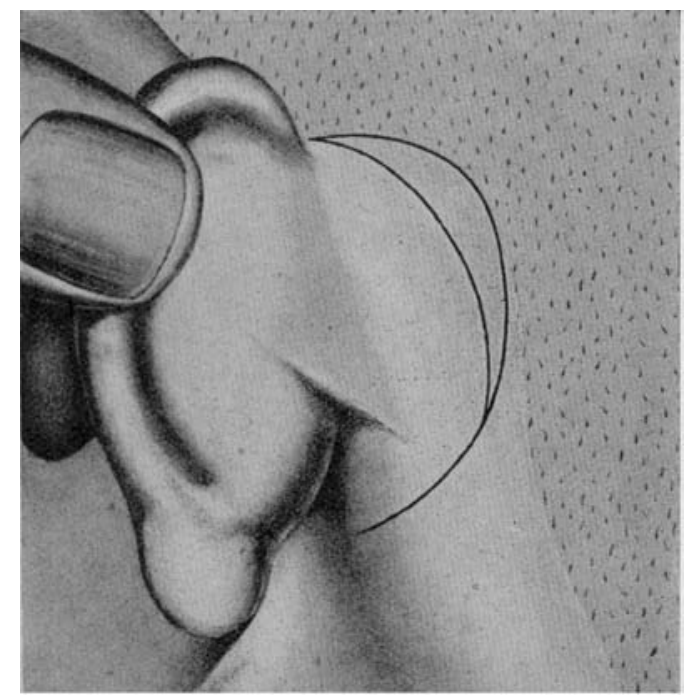

Fig. 1.-Lines of incision for outlining skin graft (left ear). The crescentic piece of skin is left attached to the periosteum, and the flap in front dissected up subcutaneously as far forward as the retro-auricular groove where the incision is made through the periosteum down to the bone.

wooden hammer is forcibly applied. The writer has had one case of facial paralysis in the early days in nearly four hundred mastoid operations. The affection almost entirely passed off within two months.

After the bridge has been removed the facial spur is very freely shaved down with a large gouge, so that the floor of the bony meatus, the spur, and the floor of the mastoid cavity form a very gentle slope (Fig. 2). Stacke's protector is never used, because if one does not feel safe without the aid of this instrument one should not operate at all. In many cases it is also advisable to level down the convexity on the floor of the bony meatus with a medium-sized gouge, so as to give good access to the tympanum for after 
treatment. It is needless to say that the external wall of the attic and aditus should also be freely removed.

In the writer's experience the lower end of the long process of the incus is usually absent in cases which come to the radical mastoid operation. As the ossicular chain is thus broken, one cannot see why the radical operation should result in any deterioration of the hearing power. In such cases the raison d'être of the modified radical operation is hard to explain. This remains true whether we look upon the chain of ossicles with its two tympanic muscles as

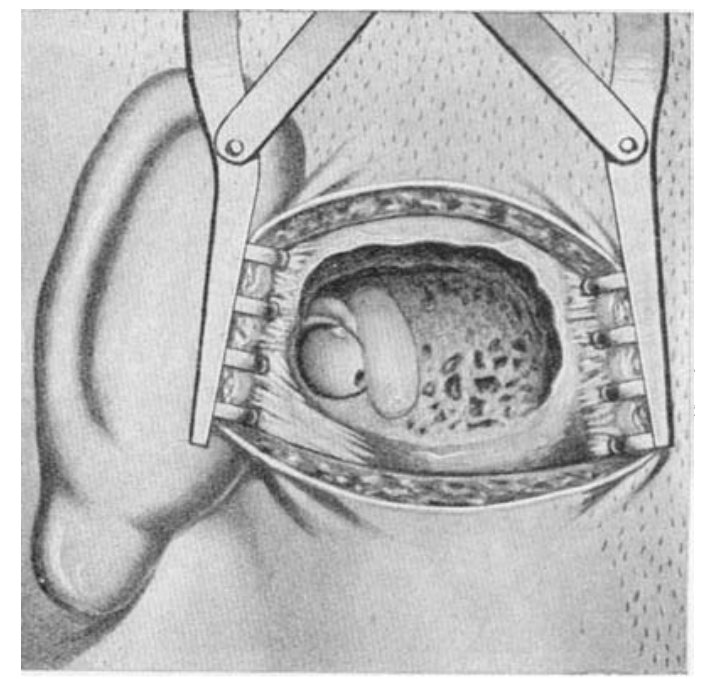

Fia. 2.-After the radical mastoid operation (left ear) bas been completed, the crescentic skin graft is cut off, trimmed, and applied over the facial spur, the inner end of the graft reaching the prominence of the external canal.

a sound-conducting apparatus or merely as a mechanism for regulating the intra-labyrinthine tension, or as both combined.

The plastic operation on the membranous external meatus may be performed at almost any period of the operation. 'The writer uses either Koerner's oblong flap or Neumann's Y flap, and performs the meatal plastic after the removal of the bridge, malleus, and incus, but before the lower part of the tympanic cavity and Eustachian tube are dealt with.

The writer believes that the inner tympanic wall should be left alone as much as possible in order not to interfere with the window niches. If, however, a polypus be present, this is impossible, as in the great majority of cases the inflammatory swelling springs from 
the lower and posterior part of the promontory between the windows. In removing such a polypus, or in curetting out with the angled spoon, the remains of the drumhead, it is most important not to scrape away the epidermis covering the anterior and inferior walls of the bony meatus in order to avoid subsequent narrowing of the meatus at the isthmus (Fig. 4). In some cases we have the formation of a complete membrane in this region, with a resulting separation of the more superficial parts of the radical mastoid cavity from the deeper portions, $i . e$. the tympanum and aditus in which suppuration may continue.

The Eustachian tube is a great difficulty. In the writer's experience there is a large class of cases of chronic suppurative otitis media, with oval perforations in the anterior part of the drumhead, or

Fig. 3.

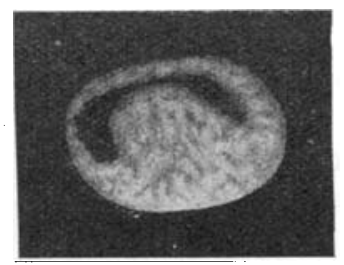

Fig. 4.

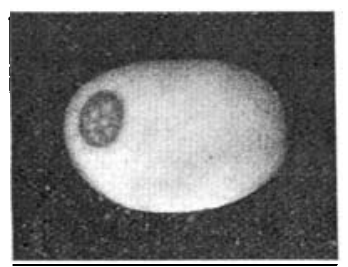

Fia. 3.-Appearance of cavity as often seen about three weeks after operation on left ear in cases in which skin graft is not applied. Granulations are seen growing upwards from the lower cut edge of the posterior bony meatal wall (facial spur), and downwards from the cut edges of the external wall of the attic and upper part of the posterior bony meatal wall. One can with difficulty see into the tympanum in front and into the antrum behind.

Fig. 4.-Appearance six to eight weeks after operation in unfavourable case. The outer part of the operation cavity has become lined by squamous epithelium, but a "contraction ring" has formed at the point where formerly the isthmus of the meatus was situated. Through this ring we can see the socalled "granulations" on the inner wall of the tympanum. In some cases the ring closes entirely, so that we have a complete formation of a membrane or partition between the outer and inner portions of the operation cavity. Suppuration may continue behind this membrane, and, if the case be again operated on some months or years later, it is found that a great deal of new bone formation has taken place.

kidney-shaped perforations below the umbo, in which the suppurative process is confined to the anterior or tubal portion of the middle ear cleft and the lower part of the tympanic cavity. The cases are really "tuborrhoeas," and in many of them the hearing power is very good. If the surgeon is ill-advised enough to perform the modified radical operation on such cases on account of the good hearing, he is disgusted to find a healthy antrum. If he now goes on to complete the radical operation so as to get good access to the Eustachian tube and tubal portion of the tympanic cavity, he is again likely to be dis- 
appointed, because he will find a large tube which is exceedingly difficult to close, even with the aid of Alexander's rectangular curettes, which are accurately made to fit the outline of the bony tube (see Jodrn. of Laryngol., RHinol., and Otol., December, 1915, p. 460, Fig. 3). It is hard to credit the claims put forward by Yankauer and other American otologists with regard to the results they obtain from curetting the Eustachian tube through the normal external auditory meatus (Laryngoscope, 1915, p. 675). Yankauer uses a small, circular curette, and advises local anæsthesia. He has collected 735 cases, operated on by various otologists. Of these, the tube was successfully closed after one or more curettings in 609 (83 per cent.), while 379 patients were reported as cured (51.5 per cent.). The writer finds it difficult to understand how surgeons, who only have the aid of local anæsthesia, who use an inefficient instrument, and operate round a corner by feel or guess-work, can obtain results which are not much worse than those following the curettage of the tube under general anæsthesia, with an efficient instrument, and guided by direct vision. Longee (Jour. Amer. Med. Assoc., 1914 , p. 1576) says the curettage of the Eustachian tube through the external meatus is of very little value. He reports twenty-one cases, and in only two instances did he obtain closure of the tube.

From what has been already said it will be seen that, in the writer's opinion, the modified radical operation is not advisable in cases of chronic middle ear suppuration (usually with a marginal perforation in the posterior superior quadrant) in which the continuity of the ossicular chain is broken by the loss of the lower end of the long process of the incus, nor in cases with anterior or central perforations associated with " tuborrhœa." In fact, the only class of case in which the modified radical operation appears to be of advantage is a comparatively small one-viz., cases of "attic" perforation with good hearing. We must, however, remember that cholesteatoma is present in most cases of attic perforation. In the writer's opinion this does not negative the modified radical operation.

In operating on cases complicated by cholesteatoma the writer does not as a rule remove the matrix, because if this be left in situ it greatly assists the rapid epidermising of the cavity. Cholesteatoma consists of squamous epithelium, and this is exactly what we hope the mastoid cavity will eventually be lined with. Perhaps a little extra care may be needed in the after-treatment of these cases, e. g. drops of salicylic acid in alcohol and more frequent syringing than usual. It is absolutely necessary to impress on all patients on whom the radical operation has been performed that they must 
have their "operated" ear attended to at least once a fortnight, and better still once a week for the rest of their lives.

In order to obtain a dry cavity the writer believes that it is very important to make a large external auditory meatus by extending the incisions for the plastic well into the concha and stitching back the flap. When the whole cavity has become thoroughly healed and the skin lining it has become pale, the increase in size of the external meatus is hardly noticeable. If the meatus be too small, the cavity tends to remain moist. This may not be a disadvantage as far as hearing is concerned, but both surgeons and patients prefer a dry ear.

The writer has found that there is not infrequently a tendency to drooping of the auricle after the radical mastoid operation, i.e. the auricle on the operated side is at a lower level than on

FIG. 5.

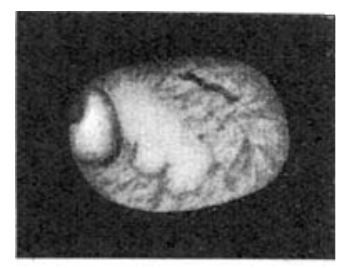

Fia. 6.

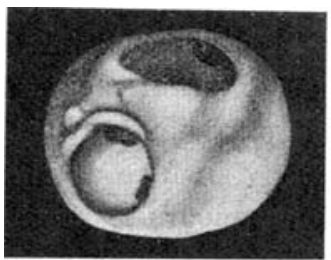

Fia. 5.-Appearance three weeks after operation (left ear) in case in which skin graft was applied to facial spur at time of operation. 'The epithelium is seen spreading over the granulations.

Fio. 6.-The result one hopes to, but does not always, see after the radical operation on the left ear. The tube is closed, and the operation cavity has healed with retention of the outline of the inner wall. The promontory, oval and round window niches, facial canal, attic, prominence of the external semicircular canal and antrum are all clearly seen. (The patient can hear a whisper at six feet with the left ear.)

the non-operated side. This is not only unsightly, but also tends to narrow the external meatus. In some cases the drooping is so marked that in order to inspect the cavity the surgeon must almost go down on his knees, or must tilt the patient's head over towards the opposite shoulder to an uncomfortable extent. To obviate this the writer has for some time removed a crescentic piece of skin at the upper and posterior part of the curved retro-auricular incision. The area removed is about 1 inch long and $\frac{1}{4}$ of an inch wide at its broadest part. For a time this small piece of skin was thrown away, but of late it occurred to the writer that the skin might be used as a graft to cover the facial spur, and so obviate the tendency to the reformation of the posterior bony wall of the external meatus. No matter how freely this wall has been removed 
above, so as to lay open the attic and aditus, and no matter how well the spur has been smoothed off below to expose the promontory and window niches, within two or three weeks after the radical operation there is, in certain cases, an exuberant growth of granulations from above and from below in the region of the cut edges of the posterior bony meatal wall (Fig. 3). Like stalactites and stalagmites, these tend to meet. This exuberant growth is especially seen in cases in which the bone is found to be very vascular at operation, and in obstinate instances no method of after treatment seems to make much difference-packing or non-packing, spirit drops or dry boric insufflations, scarlet red or liquid paraffin. Only skin-grafting is of service, but to cut and apply a skin-graft from the inner side of the thigh takes up a considerable amount of time. After trying Ballance's method of skin-grafting with reopening of the retro-auricular wound ten to fourteen days after the radical operation, the writer came to the conclusion that it was not worth while. In other cases, in which the graft was applied through the enlarged external meatus, it seldom "took." The writer has no practical experience of the application of large thin Thiersch grafts to the bony surface at the end of the operation, but a theoretical objection appears to be that the mucosa of the tympanum cannot be entirely removed, even by the most thorough curetting, e.g. in the region of the window niches, in the irregularities of the tympanic floor, in the sinus tympani, etc. It seems impossible that a skin-graft can "take" over these areas. On the other hand, grafts consisting of the whole thickness of the skin (dermis and epidermis) "take" well when applied to the raw bony surface of the facial spur and floor of the bony meatus, according to the following method: The crescentic piece of skin is outlined with the knife at the time of the original incision (Fig. 1), and, during the operation, is left attached to the periosteum covering the mastoid. When the operation is completed the cavity is syringed out with lnkewarm saline lotion and temporarily packed with sterile gauze. The crescentic portion of skin is now excised with the knife or scissors, and placed on the sterile towel covering the patient's head, so that the epidermic surface is next to the towel and the fatty subcutaneous surface upwards. The assistant now seizes one end of the graft with a pair of mouse-toothed forceps, while the operator does the same at the other end. In this way the graft is stretched between the two pairs of forceps. The subcutaneous fatty tissue is now picked up by the assistant with the aid of a third pair of forceps, while, with scissors curved on the flat, the surgeon dissects away the fatty tissue, leaving only the cutis vera and 
epidermis. The ganze is now removed from the mastoid wound and the skin-graft is applied to the cut surface of the facial spur, and covered with an oblong piece of perforated oiled silk only slightly bigger than the graft itself. If a larger crescentic piece be excised, it may be divided transversely, one portion being applied to the floor of the bony meatus which has been lowered with the gouge while the other piece is applied to the facial spur as described. The skin-grafts covered by the oiled silk are then held in position by the assistant, with the aid of a pair of angled forceps, while the surgeon packs the tympanic cavity, aditus and antrum, with iodoform worsted, which retains the graft in position after the assistant has withdrawn his forceps. The retro-auricular wound and the operation cavity are only dressed on the fifth day. Even when the iodoform worsted is removed, the oiled silk remains in position and has to be taken out with angled forceps. The skingraft invariably retains its position, and by the end of a period of ten days it is seen to be spreading out towards the antrum and also covering the floor of the meatus (Fig. 5). In one or two recent cases healing of the cavity has been complete in six weeks. The whole procedure only adds at most five minutes to the time occupied by the radical operation.

In itself the " after-treatment of the radical mastoid operation". might well form a subject for discussion. As stated above, the cavity is dressed on the fifth day and repacked. The case is again dressed on the seventh day, and daily thereafter up to the tenth. If by this time all looks well packing is omitted during the daytime, but at night, though the cavity itself is not packed, a piece of gauze is inserted into the meatus to maintain its patency and also to prevent soiling of the pillow. It must, however, be confessed that there are cases in which the "non-packing" method has to be abandoned in favour of "packing." In these cases the after-treatment is long and tedious, both to surgeon and to patient. If the latter lives in town and can attend daily at the hospital, he may be able to resume work, and, in any case, does not require to have a bed in the ward. It is different, however, in the case of country patients, whose after-treatment cannot well be left to the care of the busy practitioner or district nurse. Except for the daily treatment of the ear, these patients do not require skilled attention or nursing, and it has often struck the writer that a hospital bed was wasted in such cases. Some less elaborate form of accommodation would serve the purpose equally well. In this way hospital beds would be more quickly available for fresh operation cases, and long "waiting lists" would be largely obviated. 